\title{
The effect of aging on low-fat, reduced-fat, and full-fat Cheddar cheese texture
}

\author{
N. R. Rogers, ${ }^{*}$ M. A. Drake, ${ }^{*}$ C. R. Daubert, ${ }^{*}$ D. J. McMahon, † T. K. Bletsch, $\ddagger$ and E. A. Foegeding ${ }^{* 1}$ \\ *Department of Food, Bioprocessing, and Nutrition Sciences, North Carolina State University, Raleigh 27695 \\ †Department of Nutrition and Food Sciences, Utah State University, Logan 84322 \\ łDepartment of Computer Science, North Carolina State University, Raleigh 27695
}

\section{ABSTRACT}

This study investigated the effects of aging and fat content on the texture of Cheddar cheese, both mechanical and sensory aspects, over a 9-mo aging period. Cheeses of 6,16 , and $33 \%$ fat were tested at $0.5,3,6$, and 9 mo of aging. Cheeses were evaluated by a trained sensory panel using an established texture lexicon as well as instrumental methods, which were used to probe cheese structure. Sensory analysis showed that low-fat cheeses were differentiated from full-fat cheeses by being more springy and firm and this difference widened as the cheeses aged. In addition, full-fat cheeses broke down more during chewing than the lower fat cheeses and the degree of breakdown increased with aging. Mechanical properties were divided by magnitude of deformation during the test and separated into 3 ranges: the linear viscoelastic region, the nonlinear region, and fracture point. These regions represent a stress/strain response from low to high magnitude, respectively. Strong relationships between sensory terms and rheological properties determined in the linear (maximum compliance) and nonlinear (critical stress and strain and a nonlinear shape factor) regions were revealed. Some correlations were seen with fracture values, but these were not as high as terms related to the nonlinear region of the cheeses. The correlations pointed to strain-weakening behavior being the critical mechanical property. This was associated with higher fat content cheeses breaking down more as strain increased up to fracture. Increased strain weakening associated with an increase in fat content was attributed to fat producing weak points in the protein network, which became initiation sites for fracture within the structure. This suggests that fat replacers need to serve this functional role.

Key words: cheese, texture, rheology, low fat

Received February 23, 2009.

Accepted July 7, 2009.

${ }^{1}$ Corresponding author: allen_foegeding@ncsu.edu

\section{INTRODUCTION}

Cheese is a very popular food in the United States; the cheese industry has experienced rapid growth with product availability tripling since 1970 (Wells and Buzby, 2007). Trends toward healthier eating and a consumer preference for lower fat foods have resulted in increased interest in low-fat cheeses (Sandrou and Arvanitoyannis, 2000). However, consumer perception of low- and reduced-fat cheese has not necessarily been positive, as these cheeses often have off-flavors, different appearance, and rubbery texture (Hamilton et al., 2000; Mistry, 2001).

Lower fat cheeses are made from lower fat milks and thus have higher protein to fat ratios than fullfat cheeses; this causes the lower fat cheeses to have a more dense protein network and firmer texture. To try to compensate for the increased protein concentration, water is added back into the cheeses to offset the decreased fat content, but the texture is still firmer than full-fat cheeses (Johnson and Chen, 1995). Water addition is one of several methods used to offset fat reduction during cheese production by increasing cheese moisture; this method involves the curds being washed or soaked in water after the whey draining step (Scott, 1986; Drake and Swanson, 1995). This approach has been shown to increase consumer acceptance of lowand reduced-fat cheeses (Drake et al., 1995).

The aging or ripening of cheese contributes to the final texture because of the chemical and structural changes that happen over time, which are primarily related to the hydrolysis of proteins. This degrades the casein network over time and results in a less firm and more deformable cheese (Tunick et al., 1990; Banks, 2007). Proteolysis occurs in 2 stages, the initial stage being within the first $14 \mathrm{~d}$ of aging when around $20 \%$ of the casein in the network is hydrolyzed, and a second period occurs during long-term aging, when a more gradual breakdown takes place (Lawrence et al., 1987). Lawrence et al. (1987) also implied that moisture present in the cheese had an effect on texture, suggesting that as proteolysis progresses, and the peptide bonds 
are broken, ionic groups are exposed and tie up the free water in the cheese, increasing cheese firmness.

Consumer perception of a cheese is composed of several factors, including visual aspects, flavor, and texture. A trained sensory texture panel is a valuable tool in understanding what aspects of texture differentiate cheese (Foegeding and Drake, 2007). Descriptive sensory panels and texture terminology (lexicon) specifically designed for cheese have been used in the past to probe the texture attributes of many varieties of cheese (Drake et al., 1999a; Brown et al., 2003; Carunchia Whetstine et al., 2007; Yates and Drake, 2007). The established texture lexicon comprises 3 parts: tactile response, first bite, and breakdown during chewing (Brown et al., 2003; Foegeding and Drake, 2007). The use of a consistent descriptive lexicon of terms provides a "textural fingerprint" for a cheese that not only allows comparison with other cheeses analyzed with the same lexicon and standards, but also facilitates correlation with rheological properties.

Although sensory evaluation is the main method for determining perception of cheese texture, fundamental rheological tests are also needed to understand why these texture differences exist. These tests are crucial as they provide a connection to established mechanisms based on molecular and microstructural considerations (Foegeding et al., 2003). Linking mechanical properties and oral processing with sensory perception of texture would allow for a rational design of texture (Foegeding, 2007). Many studies in the past have used mechanical texture profile analysis (TPA) in combination with sensory analysis (Lee et al., 1978; Casiraghi et al., 1989; Bryant et al., 1995). Although these studies have shown correlations between sensory and TPA terms, TPA was designed to be a mechanical imitator of sensory analysis, and thereby provides no description of structural elements. In contrast, fundamental rheological tests measure properties that can be linked to structural elements in cheeses (Foegeding et al., 2003).

Fracture properties of cheese (fracture stress and strain) have been determined under compression (normal force; Jack et al., 1993; Hort et al., 1997; Hort and Grys, 2000). Tests that apply a normal (compressive) force to cause fracture correlate well with sensory lexicon terms related to firmness (Drake et al., 1999b). Tests have also been conducted in torsion, in which the sample is twisted rather than compressed until fracture (Diehl and Hamann, 1979). The stress and strain at fracture, determined by normal or torsional deformation, have been shown to correlate with sensory springiness and firmness (Drake et al., 1999b; Gwartney et al., 2002; Brown et al., 2003; Carunchia Whetstine et al., 2007). This is logical in that sensory and mechani- cal tests are evaluating the initial breakdown of the cheese.

Alternatively, determining mechanical properties of a material without damaging it is often desirable to probe the basic nature of a material. Small-strain rheological tests can achieve this goal and are conducted within the linear viscoelastic region (LVR). This means that material properties such as complex modulus $\left(\mathbf{G}^{*}\right)$, stress applied divided by the strain measured or vice versa for each oscillation, are not dependent on level of stress or strain (Steffe, 1996). These small-strain properties have been shown to correlate well with certain sensory terms relating to the breakdown of the cheese during mastication as well as the firmness of both hand evaluation and during the first bite of the sample, but these correlations often fail to be consistent from study to study (Drake et al., 1999c; Brown et al., 2003). Brown et al. (2003) suggests that this lack of consistent correlations can be attributed to small-strain properties not reflecting mechanical properties the sensory panelists detect during mastication.

Measuring the deformation response to a prolonged applied force is useful in understanding viscoelastic behavior. Previous studies have used creep/recovery tests to understand this behavior and relate it to sensory panel data. Values for maximum compliance, retardation time (measure of the viscoelastic nature of a material), and percentage recovery (recovery relative to the maximum deformation after the initial force is released) have all been shown to correlate strongly with sensory terms related to firmness and springiness (Steffe, 1996; Brown et al., 2003). However, sensory terms such as cohesiveness and smoothness, and other terms related the breakdown of the cheese during chewing, are weakly or not correlated with mechanical properties (Brown et al., 2003). Because these terms involved tactile and adhesive properties during mastication, it was believed that additional rheological testing involving mechanical adhesion would correlate with these sensory terms better.

To our knowledge, previous studies have not taken a complete approach to evaluation of the effect of fat reduction in Cheddar cheeses: 1) cheeses were not manufactured for maximum experimental control, 2) a full range of commercially relevant fat contents were not evaluated, or 3) both sensory and rheological changes in texture with ripening time and fat reduction were not compared. The purpose of this study was to determine textural differences in cheeses with differing contents of fat and how textural properties were affected by aging. This study was part of a multi-university investigation on low-fat cheese. Cheese manufacturing and chemical analysis was conducted at Utah State University. Cheeses were then shipped to North Carolina State 
Table 1. Cheese manufacturing procedures used for making full-fat, $50 \%$ reduced-fat, and low-fat cheeses

\begin{tabular}{|c|c|c|c|c|}
\hline \multirow[b]{2}{*}{ Item } & \multicolumn{4}{|c|}{ Cheese $^{1}$} \\
\hline & FF1 & FF2 & $\mathrm{RF}$ & LF1/LF2 \\
\hline Ripening time (min) & 45 & 45 & 45 & 45 \\
\hline Set temperature $\left({ }^{\circ} \mathrm{C}\right)$ & 31 & 31 & 31 & 31 \\
\hline Annatto $(\mathrm{mL} / 100 \mathrm{~kg}$ of milk $)$ & 13 & 13 & 13 & 13 \\
\hline Rennet (mL/100 kg of milk) & 9 & 9 & 9 & 9 \\
\hline Set-to-cut time (min) & 30 & 30 & 30 & 25 \\
\hline Curd size & Small & Small & Large & Large \\
\hline Set-to-start of cook time (min) & 50 & 50 & 60 & 50 \\
\hline Cooking temperature (time), ${ }^{\circ} \mathrm{C}(\min )$ & $37(25)$ & $37(25)$ & $37(20)$ & $36(15)$ \\
\hline Set-to-drain time (min) & 150 & 150 & 150 & 100 \\
\hline Drain $\mathrm{pH}$ & 6.0 & 6.0 & 6.0 & 6.0 \\
\hline Wash water temperature $\left({ }^{\circ} \mathrm{C}\right)$ & $\mathrm{NA}^{2}$ & 35 & 14 & 14 \\
\hline Curd wash temperature $\left({ }^{\circ} \mathrm{C}\right)$ & NA & 36 & 26 & 26 \\
\hline Set-to-salt time $(\min )$ & 190 & 190 & 185 & 185 \\
\hline Salting $\mathrm{pH}$ & 5.45 & 5.45 & 5.75 & 5.80 \\
\hline Salt $(\mathrm{g} / \mathrm{kg}$ of curd) & 24 & 24 & 25 & 26 \\
\hline Pressing $(\mathrm{kPa})$ & 100 & 100 & 55 & 55 \\
\hline
\end{tabular}

${ }^{1}$ Cheeses included 2 full-fat cheeses (FF1 and FF2) that differed only in inclusion of a curd washing while making FF2, 1 reduced-fat (RF) cheese, and 2 low-fat cheeses (LF1 and LF2) that differed only in the starter culture used.

${ }^{2} \mathrm{NA}=$ not analyzed.

University at each time point for sensory and rheological analyses. The specific goals of this research were to characterize the sensory and rheological differences among Cheddar cheeses at different fat contents as they aged, and to see if these differences could be related to structural aspects of the cheese network.

\section{MATERIALS AND METHODS}

\section{Cheddar Cheese Production}

Fresh cow's milk from the Utah State University's Caine Dairy Research and Teaching Center (Wellsville) and transported to the Gary Haight Richardson Dairy Products Laboratory (Logan, UT). Milk was standardized to protein to fat ratios of $0.83,1.9$, and 5.0 for making full-fat $(\mathbf{F F}), 50 \%$ reduced-fat $(\mathbf{R F})$, and lowfat $(\mathbf{L F})$ cheeses, respectively, and then pasteurized at $73^{\circ} \mathrm{C}$ for $15 \mathrm{~s}$. When making LF cheese the milk $(682$ $\mathrm{kg}$ ) was cooled to $10^{\circ} \mathrm{C}$, poured into a Tetra Scherping horizontal cheese vat (Tetra Pak Cheese and Powder Systems Inc., Winsted, MN), and then acidified to $\mathrm{pH} 6.25$ with distilled white vinegar (Heinz North America, Pittsburgh, PA) and then warmed to the set temperature. Five different cheeses (in duplicate) were made in randomized order. This included $2 \mathrm{FF}$ cheeses (FF1 and FF2) that differed only in inclusion of a curd washing while making FF2, 1 RF cheese, and $2 \mathrm{LF}$ cheeses (LF1 and LF2) that differed only in the starter culture used. All of the cheeses except LF2 used a single strain Lactococcus lactis ssp. lactis starter culture (M70, Danisco USA Inc., New Century, KS), whereas LF2 was made using a blend of Lactococcus lactis ssp. lactis/ cremoris (DVS850) starter culture along with a Lac ${ }^{-} L c$. lactis culture (CR319) and a Lactobacillus helveticus culture (LH 32) all from Chr. Hansen Inc. (Milwaukee, WI). Double-strength chymosin (Maxiren) and singlestrength annatto cheese color were from DSM Food Specialties USA Inc. (Eagleville, PA). The cheeses were made following procedures outlined in Table 1. Some of the cheese-making parameters were adjusted to allow the texture of the RF and LF cheeses to better match that of currently available commercial cheeses with similar levels of fat. These modifications led to the RF and LF cheeses having higher moisture content than the FF cheeses. Although the moisture level was varied, the ratio between protein and moisture was held constant in all of the cheeses. The cheeses were stored at $8^{\circ} \mathrm{C}$ for 9 mo with sample blocks shipped to North Carolina State University for texture testing at $0.5,3$, 6 , and 9 mo of age.

\section{Rheological Analysis}

Determination of the $\boldsymbol{L V R}$. Stress sweeps of cheeses were done using a Stress Tech controlled stress rheometer (ATS Rheosystems, Bordentown, NJ) fitted with a $20-\mathrm{mm}$ smooth parallel plate geometry. Temperature was controlled using an integrated induction heating device set at $25^{\circ} \mathrm{C}$. Cheese samples were sliced to a thickness of $4 \mathrm{~mm}$ and trimmed to the size of the plate. Samples were glued to both plates to prevent slip using cyanoacrylate glue (Loctite 401, Loctite Corp., Rocky Hill, CT). Once glued, the exposed edges of the sample had a thin layer of synthetic lubricant applied (SuperLube, Synco Chemical, Bohemia, NY) to 
prevent sample dehydration during testing. Tests were conducted at $10 \mathrm{~Hz}$ with a stress range from 1 to 1,000 $\mathrm{Pa}$ on each cheese for each replication. The critical stress and strain were determined as the point when $\mathrm{G}^{*}$ values decreased consistently; the stress and strain on the sample at that point were labeled the critical stress and critical strain.

Creep/Recovery Analysis. Creep/recovery tests were conducted using a Stress Tech controlled stress rheometer (ATS Rheosystems). Tests were conducted at 100 and $150 \mathrm{~Pa}$ and forces were applied to the sample for $200 \mathrm{~s}$, removed, and the recovery of the sample was measured for an additional $200 \mathrm{~s}$. Tests at each force were conducted in duplicate for each replication at $25^{\circ} \mathrm{C}$. The values of maximum compliance $\left(\mathbf{J}_{\max }\right)$, instantaneous compliance $\left(\mathrm{J}_{0}\right)$, retardation time $\left(\lambda_{\text {ret }}\right)$, and percentage recovery (crp) were determined from the creep/recovery data. Maximum compliance was the maximum deformation reached before the force was removed. Instantaneous compliance was the compliance as time approached zero. Retardation time was calculated as the time for the strain to reach $63.2 \%$ of its final value. The percentage recovery was calculated using the equation

$$
\operatorname{crp}=\frac{\left(J_{\max }\right)-\left(J_{r}\right)}{J_{\max }},
$$

where $J_{\max }$ is the maximum compliance and $J_{r}$ is the compliance after the sample has been allowed to fully recover (Brown et al., 2003).

Large-Strain Torsion Analysis. Nonlinear and fracture analysis were conducted using the torsion method adapted from Brown et al. (2003). Cheese blocks were held at room temperature in sealed plastic bags for $8 \mathrm{~h}$ to allow them to come to room temperature $\left(25^{\circ} \mathrm{C} \pm 4^{\circ} \mathrm{C}\right)$. Cheese samples were taken from sample blocks using an 18-mm-diameter cork borer and cut to a length of $28 \mathrm{~mm}$. These samples had notched, plastic disks (Gel Consultants, Raleigh, NC) glued to each end of the sample using cyanoacrylate glue (Loctite 401, Loctite Corp.). Samples were then ground into a capstan shape using a precision grinding machine (model GCPM92 US, Gel Consultants). Samples were tested using a Haake VT-550 rotational viscometer (Gerbruder Haake GmbH, Karlsruhe, Germany) fitted with an attachment designed to facilitate torsion testing (Truong and Daubert, 2000). Five samples were tested for each replication at 3 strain rates, 0.040, 0.40, and $4.0 \mathrm{~s}^{-1}$ (i.e., total of 15 samples per treatment per replication).
The torsion test measured torque and time during twisting until the materials fractured. The point at which the material fractured was determined as the point (torque and time) where the torque values began to decrease consistently. These values were used to calculate stress $(\sigma)$ and true strain $\left(\gamma_{\text {true }}\right)$ at fracture using the following equations (Nadai, 1937; Diehl and Hamann, 1979; Hamann, 1983):

$$
\begin{gathered}
\sigma=\frac{2 K M}{\pi r_{\min }^{3}}, \\
\gamma_{t}=\frac{2 K \varphi}{\pi r_{\min }^{3} Q}, \text { and } \\
\gamma_{\text {true }}=\ln \left[1+\frac{\gamma_{t}^{2}}{2}+\gamma_{t}\left(1+\frac{\gamma_{t}^{2}}{4}\right)^{1 / 2}\right],
\end{gathered}
$$

where $K$ is a shape factor constant, 1.08; $M$ is the torque value from the torsion test; $r_{\min }$ is the radius at the minimum of the capstan-shaped sample and was used to calculate stress ( $\sigma$; Nadai, 1937; Diehl and Hamann, 1979). True strain $\left(\gamma_{\text {true }}\right.$, hereafter referred to as strain) was calculated using the uncorrected strain value $\left(\gamma_{t}\right)$, which was calculated using angular deformation of the sample $(\varphi)$ and a curvature section constant $(8.45 \times$ $10^{-6} \mathrm{~m}^{-3}$ ) $Q$ (Nadai, 1937; Diehl and Hamann, 1979). Fracture modulus $\left(G_{f}\right)$ was calculated using the shear stress and strain at the fracture point (Brown et al., 2003):

$$
G_{f}=\frac{\sigma}{\gamma_{\text {true }}} .
$$

The nonlinear region of the fracture curves was analyzed using a nonlinear curve fitting technique, which has been used in previous studies to model the nonlinear region, using a strain energy density function developed by Blatz, Sharda, and Tschoegl (BST; Blatz et al., 1974; Barrangou et al., 2006a):

$$
\begin{gathered}
\sigma=\frac{2 G_{B S T}}{n}\left(\lambda^{n}-\lambda^{-n}\right) \text { and } \\
\lambda=\frac{\gamma+\left(\gamma^{2}+4\right)^{1 / 2}}{2},
\end{gathered}
$$


Table 2. Descriptive texture lexicon for cheese developed by Brown et al. (2003) with some modification

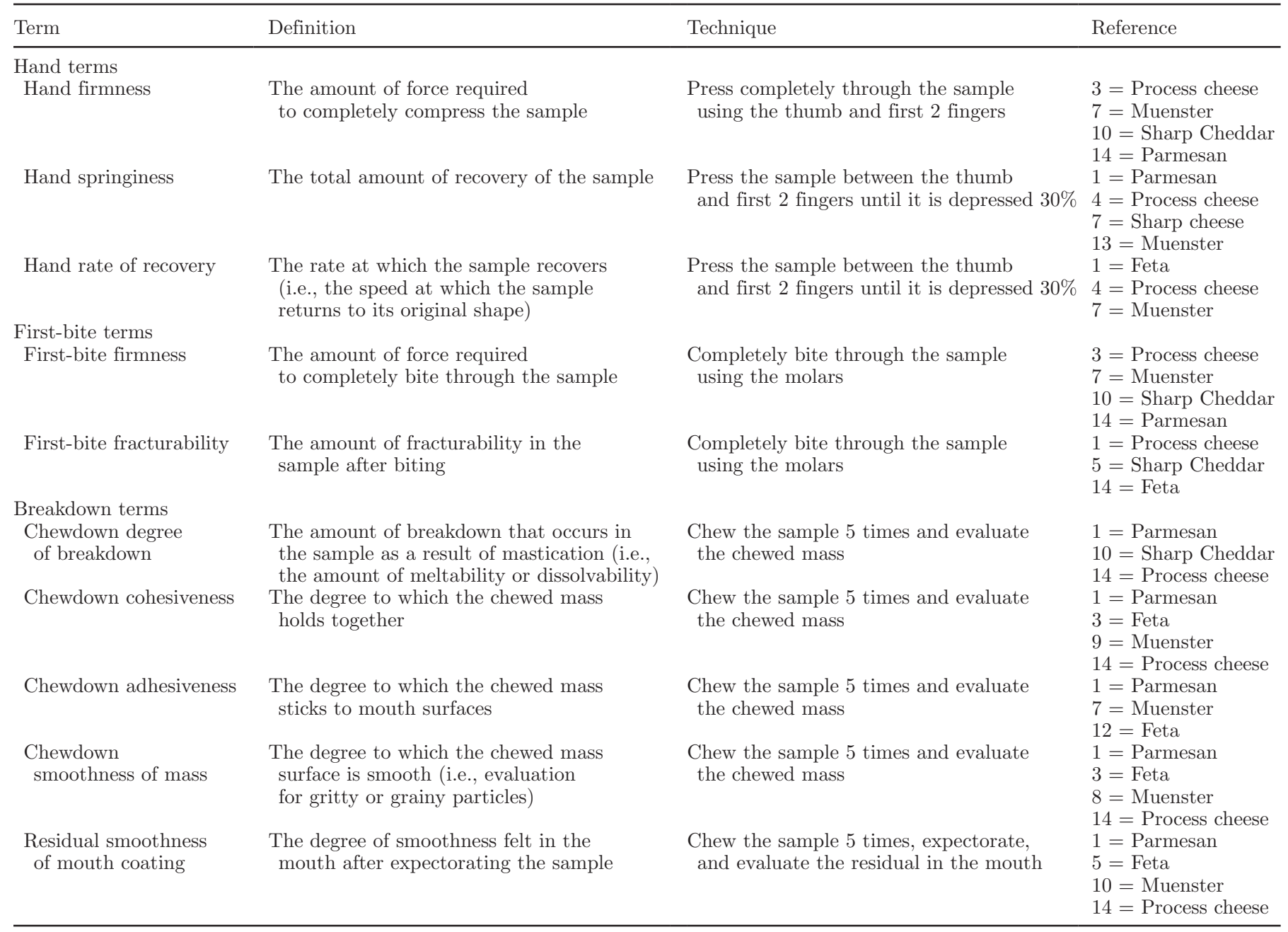

where $\boldsymbol{G}_{\boldsymbol{B} S \boldsymbol{T}}$ refers to the initial slope of fracture curve, $n$ is a curve shape constant referring to its deviation from the ideal, and $\lambda$ is a stretch ratio (Barrangou et al., 2006a). Stress and strain between time zero and fracture were used as the data to fit the model. The equation was fit to the data using the Taylor-NewtonGauss method for nonlinear regression and output values of $G_{B S T}$ and $n$ were compiled. The fitted $G_{B S T}$ was compared with the initial slope $\left(\boldsymbol{G}_{\text {initial }}\right)$ of the torsion curve; $G_{\text {initial }}$ was calculated by fitting a linear equation to the first 5 data points of each torsion curve and determining the slope (Barrangou et al., 2006a).

Instrumental Adhesion Testing. Instrumental surface adhesion of the cheese was conducted using a Brookfield LFRA texture analyzer (Brookfield Engineering, Middleboro, MA) with an attached dental

Table 3. Main effects (age and fat) and interaction (combined age and fat) for fracture stress, strain, and modulus for Cheddar cheese ${ }^{1}$

\begin{tabular}{|c|c|c|c|c|c|c|c|c|c|}
\hline \multirow[b]{3}{*}{ Item } & \multicolumn{9}{|c|}{ Strain rate } \\
\hline & \multicolumn{3}{|c|}{$0.04 \mathrm{~s}^{-1}$} & \multicolumn{3}{|c|}{$0.4 \mathrm{~s}^{-1}$} & \multicolumn{3}{|c|}{$4 \mathrm{~s}^{-1}$} \\
\hline & Stress & Strain & Modulus & Stress & Strain & Modulus & Stress & Strain & Modulus \\
\hline Age & $*$ & - & - & $*$ & - & $*$ & $*$ & - & $*$ \\
\hline Fat & $*$ & $*$ & $*$ & - & - & - & - & $*$ & - \\
\hline Interaction & - & - & - & $*$ & - & - & - & - & - \\
\hline
\end{tabular}

${ }^{1}$ Asterisk $\left({ }^{*}\right)$ denotes presence of an effect at $P<0.05$; dash $(-)$ denotes nonsignificant effect $(P>0.05)$. 


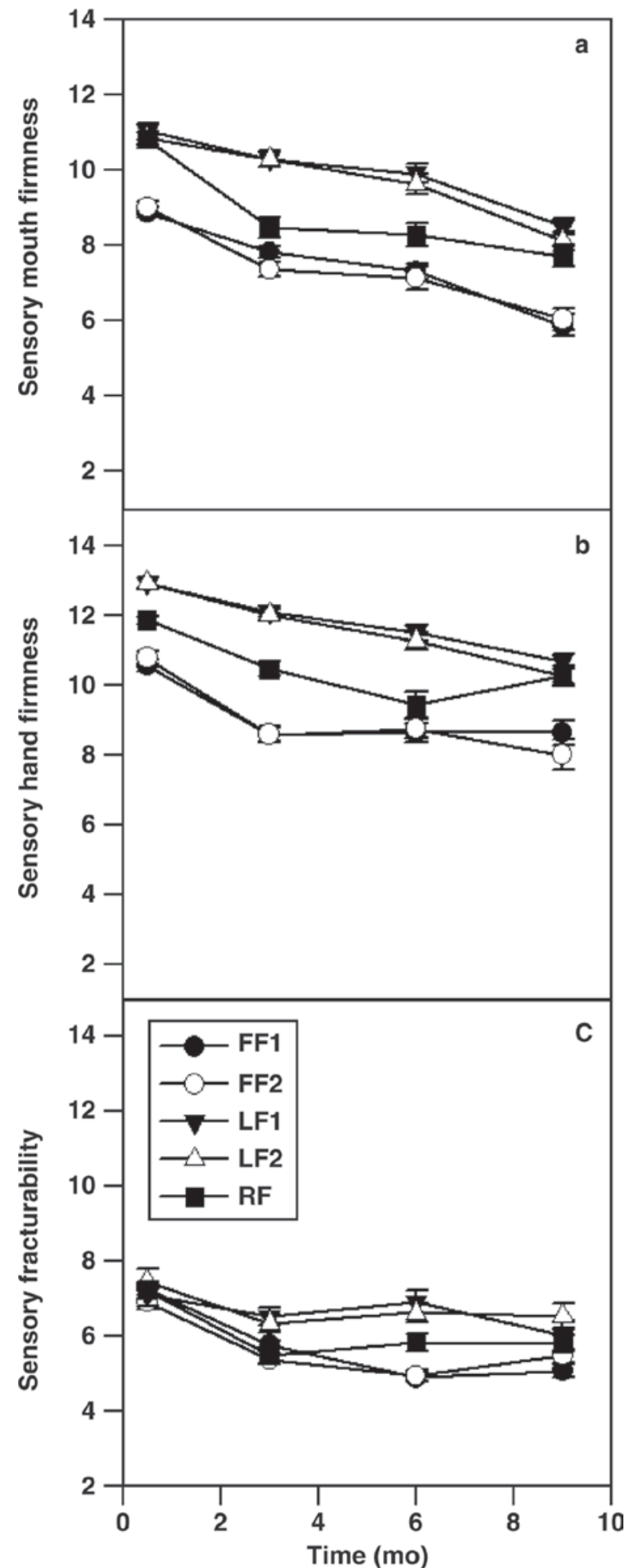

Figure 1. Changes in firmness and first-bite sensory terms of a) mouth (first-bite) firmness, b) hand firmness, and c) first-bite fracturability. Error bars represent the standard error of the mean. Cheeses included 2 full-fat cheeses (FF1 and FF2) that differed only in inclusion of a curd washing while making FF2, 1 reduced-fat (RF) cheese, and 2 low-fat cheeses (LF1 and LF2) that differed only in the starter culture used. composite probe with a circular contact surface $12.5 \mathrm{~mm}$ in diameter utilizing a modified method of Steiner et al. (2003). Sample blocks were held at room temperature in sealed plastic bags for $8 \mathrm{~h}$ to allow them to come to room temperature $\left(25^{\circ} \mathrm{C} \pm 4^{\circ} \mathrm{C}\right)$. Cheese samples were cut into cubes of $17 \mathrm{~mm} \times 17 \mathrm{~mm} \times 17 \mathrm{~mm}$ to provide a fresh surface for adhesion. Tests were conducted at a probe speed of $0.1 \mathrm{~mm} / \mathrm{s}$ and samples were loaded with a force of $200 \mathrm{~g}$. The force was held on the sample for $5 \mathrm{~s}$ and then removed at the same rate. Five samples from each replication were tested and area under the adhesion curve was determined. This was calculated by taking the absolute value of all forces less than zero and integrating them using Simpson's rule.

\section{Sensory Analysis}

Descriptive sensory analysis was conducted using the methods of Brown et al. (2003) and Yates and Drake (2007). Analysis was conducted using an experienced texture panel ( 7 females, ages $46-64 \mathrm{yr}$ ). Each panelist had approximately $200 \mathrm{~h}$ of experience in descriptive texture analysis of cheeses utilizing the Spectrum method on a 0 - to 15-point scale with a product-specific, established cheese texture language (Brown et al., 2003; Yates and Drake, 2007). This lexicon is made up of texture terms: hand firmness, hand springiness, hand rate of recovery, first-bite firmness, first-bite fracturability, chewdown degree of breakdown, chewdown cohesiveness, chewdown adhesiveness, chewdown smoothness of mass, and residual smoothness of mouth coating (Table 2; Brown et al., 2003). Panelists were provided with 8 cubes $\left(1.27 \mathrm{~cm}^{3}\right)$ of each cheese, at room temperature $\left(25^{\circ} \mathrm{C} \pm 4^{\circ} \mathrm{C}\right)$, to be used throughout testing at the discretion of the panelist in lidded, 118-mL plastic cups labeled with 3-digit codes. Panelists were given deionized water to cleanse their palates between each sample and reference cheeses were made available for each session. Samples were evaluated in triplicate by each panelist for each replication. Products were evaluated using paper ballots or Compusense Five, version 4.6 (Compusense Inc., Guelph, Ontario, Canada).

\section{Statistical Analysis}

All statistical analysis was conducted using SAS statistical software (version 9.1, SAS Institute Inc., Cary, NC). Sensory and rheological data were evaluated using a mixed model (PROC MIXED) to explore effects due to aging, fat content, and the combined effects from these variables. Fat content was used as the fixed effect in the model. Relationships among sensory terms and rheological values were determined using correlation analysis (PROC CORR). 


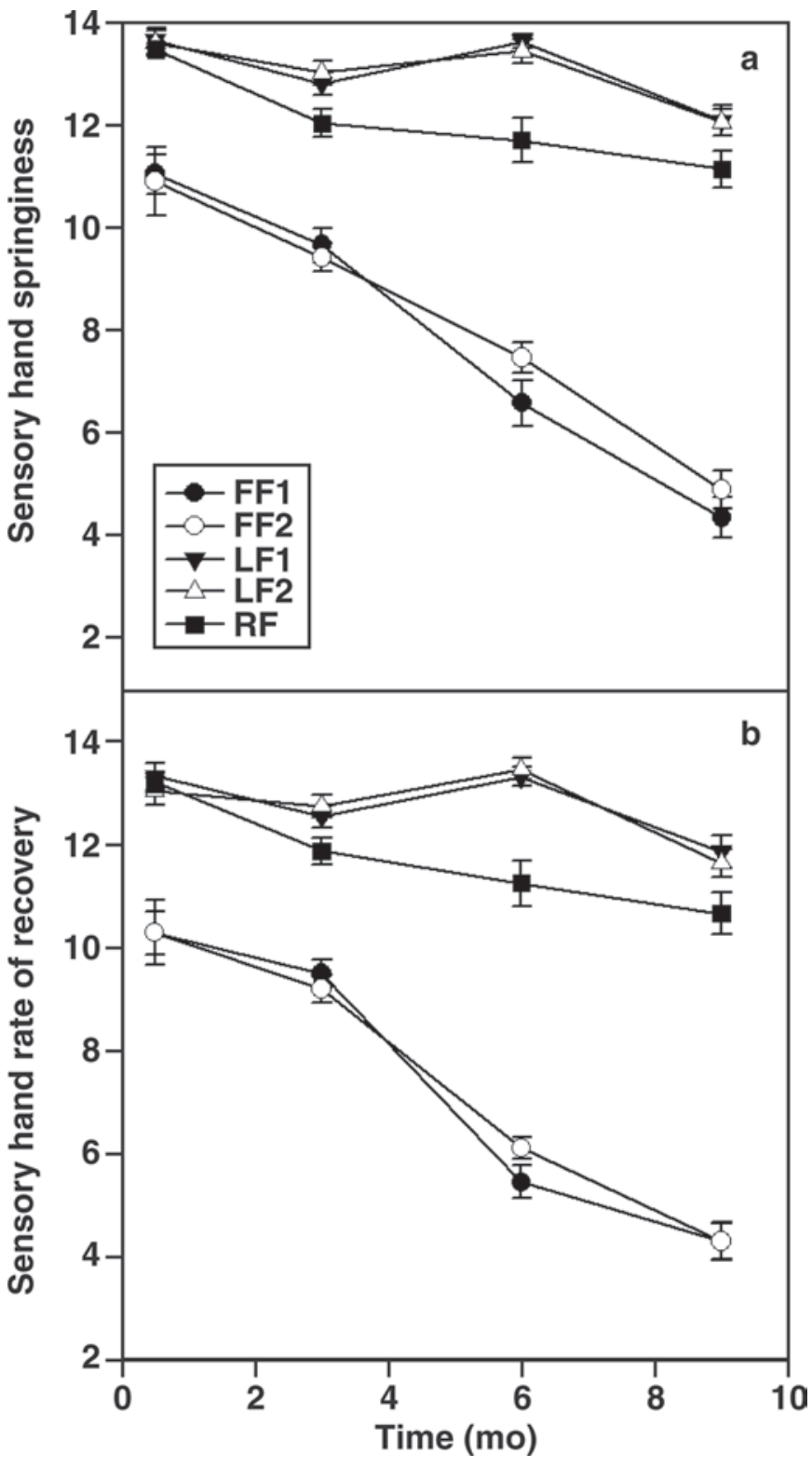

Figure 2. Changes in hand measured sensory terms of a) hand springiness and b) hand rate of recovery. Error bars represent the standard error of the mean. Cheeses included 2 full-fat cheeses (FF1 and FF2) that differed only in inclusion of a curd washing while making FF2, 1 reduced-fat (RF) cheese, and 2 low-fat cheeses (LF1 and LF2) that differed only in the starter culture used.

\section{RESULTS AND DISCUSSION}

\section{Statistical Analysis of Effects Caused by Aging and Fat Content}

All of the sensory terms were differentiated by age $(P$ $<0.05)$ and all except residual smoothness of mouth coating were differentiated by fat content $(P<0.05)$. There were age and fat content interactions $(P<0.05)$ for all terms except mouth and hand firmness and first- bite fracturability, meaning that the effects of age and fat content were interrelated for all but these terms. Results for linear and nonlinear tests of mechanical properties showed an effect $(P<0.05)$ related to fat content for critical stress and strain, an aging effect $(P<0.05)$ with $\mathrm{J}_{\max }$ and $n$, no effects with $G_{B S T}$, and both age and fat content effects $(P<0.05)$ associated with adhesive force. None of these tests showed interactions $(P>0.05)$. Torsional fracture main effects are summarized in Table 3 for fracture stress, strain, and modulus. Different strain rates are presented to cover a range of viscous relaxations during testing. It appeared that the slowest strain rate $\left(0.04 \mathrm{~s}^{-1}\right)$ was the most discriminating.

\section{Descriptive Sensory Terms}

Sensory texture terms were grouped into 3 categories based on level of deformation/mastication applied during testing. These groupings are supported by correlations among sensory and mechanical terms demonstrated by Brown et al. (2003). Sensory terms for firmness, both first-bite (Figure 1a) and hand evaluation (Figure 1b), as well as first-bite fracturability (Figure 1c), were grouped together because they were all evaluated during the initial deformation to fracture. These terms all showed similar changes as the cheese aged, with a high initial decrease between 0.5 and 3 mo, followed by a more gradual decrease. This decrease in firmness was expected as the proteolysis that occurs during the aging of cheese has been shown to decrease firmness ( $\mathrm{Tu}-$ nick et al., 1990). The higher initial change in firmness terms between 0.5 and 3 mo has been attributed to the higher rate of proteolysis during the first $14 \mathrm{~d}$ of aging (Lawrence et al., 1987). These differences were not seen consistently in all of the cheeses for this study, with the FF cheeses showing this behavior, the LF cheeses showing a steady decrease across all time points, and the $\mathrm{RF}$ cheeses showing behavior that was a combination of the 2 trends.

The sensory terms hand springiness (Figure 2a) and hand rate of recovery (Figure $2 \mathrm{~b}$ ) were grouped together as they both probe the cheese at nonfracture deformations (30\% of initial height) and thereby relate to the elastic or plastic nature of a material (Steffe, 1996). These terms showed clear distinctions between $\mathrm{FF}, \mathrm{RF}$, and LF cheeses, with the $2 \mathrm{FF}$ cheeses grouped closely together and the $2 \mathrm{LF}$ cheeses also grouped the same. All cheeses decreased in hand springiness and hand rate of recovery over time, but the decrease was lower for the LF compared with FF cheeses. The FF cheeses, on the other hand, lost much of their initial springiness consistently as they aged. The RF cheese showed behavior in between the FF and LF cheeses. 
Table 4. Proximate analysis data for Cheddar cheeses of varying fat contents

\begin{tabular}{lccccc}
\hline Cheese $^{1}$ & $\begin{array}{c}\text { Fat } \\
\text { percentage (\%) }\end{array}$ & $\begin{array}{c}\text { Moisture } \\
\text { content (\%) }\end{array}$ & $\begin{array}{c}\text { Moisture to } \\
\text { protein ratio }\end{array}$ & $\begin{array}{c}\text { Protein } \\
\text { content (\%) }\end{array}$ & $\mathrm{pH}$ \\
\hline FF1 & 31.8 & 37.7 & $1.5: 1$ & 24.6 & 5.2 \\
FF2 & 32.0 & 37.5 & $1.5: 1$ & 24.7 & 5.2 \\
RF & 15.9 & 48.0 & $1.6: 1$ & 29.6 & 5.2 \\
LF1 & 5.0 & 53.8 & $1.5: 1$ & 34.2 & 5.2 \\
LF2 & \multicolumn{2}{c}{ Not analyzed } \\
\hline
\end{tabular}

${ }^{1}$ Cheeses included 2 full-fat cheeses (FF1 and FF2) that differed only in inclusion of a curd washing while making FF2, 1 reduced-fat (RF) cheese, and 2 low-fat cheeses (LF1 and LF2) that differed only in the starter culture used.

This suggests that the fat content has a significant effect on the structure of the protein-fat network. Reducing fat in the cheese resulted in an increase in total protein and moisture (Table 4); however, the moisture to protein ratio remained consistent among cheeses at 1.5 or 1.6:1. Therefore, the protein gel network composition surrounding the fat particles was consistent among treatments. This allows the cheeses to be described using the filled gel model, where the filler (fat) in the cheese is being removed and replaced by more gel network (Visser, 1991). Lower fat cheeses would have a more homogeneous and connected network than full-fat cheeses, with fewer structural inhomogeneities caused by the fat. This more connected structure could be why the lower fat cheeses had higher initial springiness and recovery.

The breakdown terms (Table 2) were grouped together because they showed similar trends and are intercorrelated (Drake et al., 1999a; Brown et al., 2003). To reduce redundancy, the word "chewdown" was removed from the terms in figures and subsequent discussion. All breakdown terms had similar separations based on fat content as the other sensory terms, with the 2 FF cheeses grouped together, the $2 \mathrm{LF}$ cheeses being grouped, and the RF in between (Figure 3). All cheeses showed the same high initial change between 0.5 and 3 mo followed by a more gradual increase, similar to what was observed for the firmness terms. Cheeses started at approximately the same level for each of these terms, with the $2 \mathrm{FF}$ cheeses starting slightly higher than the $\mathrm{RF}$ and LF cheeses. Within the first 3 mo, differences in breakdown between the fat levels became apparent, and these differences persisted throughout the rest of the aging. The sensory terms are interrelated as the degree of breakdown of a material determines the particle size distribution, and particle size is related to cohesiveness, adhesiveness, and the residual smoothness of mouth coating because of differences in surface area of the particles. Higher adhesiveness results in greater mouth coating, which translates to greater residual smoothness of mouth coating (Brown et al., 2003).
Overall, texture properties of LF Cheddar cheeses did not change as much over a 9-mo period compared with FF cheeses. The textures of LF cheeses were firm and springy initially and did not lose springiness over time compared with the FF cheeses. In addition, FF cheeses broke down more as they aged, becoming more cohesive and adhesive than the LF cheeses. These results provide valuable insight into how the cheeses vary, but to understand why these differences exist, structural analysis through rheological measurements was also conducted.

\section{Viscoelastic Properties}

Stress Sweeps. Critical strain (Figure 4a) and stress (Figure 4b) values from LVR determination provide the point where a material deviates from its linear stress and strain relationship; these points show where network properties of the material change on a nano or micro scale. This test probed the cheese structure on a small time scale (one cycle is completed in $0.1 \mathrm{~s}$ ) and thus determined the instantaneous response. Critical stress and strain separated cheeses into 2 groups: FF versus $\mathrm{RF}$ and $\mathrm{LF}$ combined. The FF cheeses had lower critical stress and strain values that did not change with aging. This means that the FF cheeses structure broke down at a lower force and deformation compared with their LF and RF counterparts. In other words, a small force or deformation caused a weakening of the structure. The LF and RF cheeses had higher critical stresses and strains that changed with aging, but there was no constant trend with aging. Although the precise changes in cheese structure cannot be clearly seen from rheological information alone, it is clear that the elements being probed are different between the groups of cheeses.

Creep/Recovery. Values for $\mathrm{J}_{\max }$ are shown in Figure 5. In this test, the stresses and strains are maintained within the LVR, but the longer testing time (200 s) allows for a greater contribution from viscous effects. The LF cheeses had higher $\mathrm{J}_{\max }$ over time, which means 

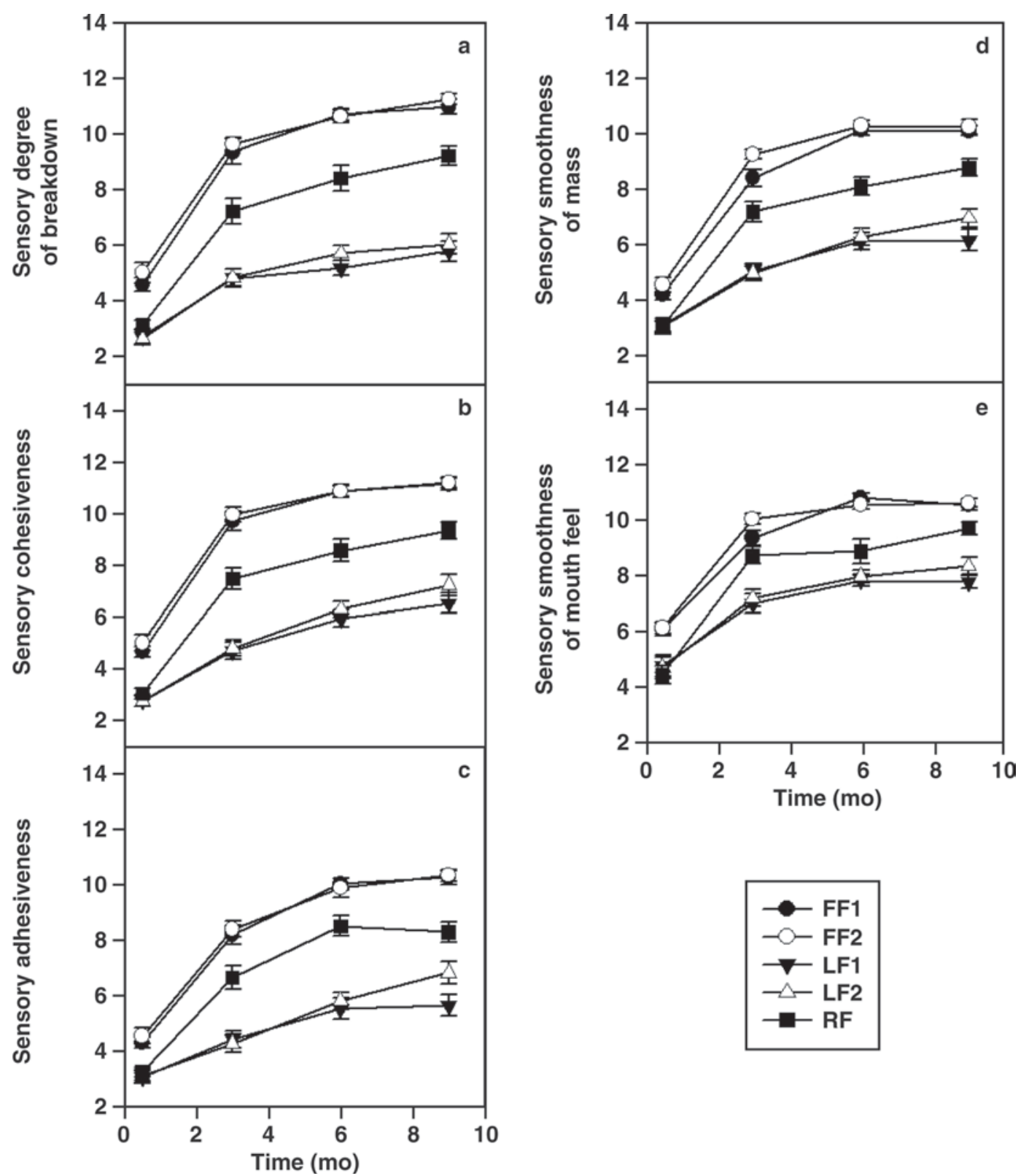

Figure 3. Changes in breakdown terms of a) degree of breakdown, b) cohesiveness, c) adhesiveness, d) smoothness of mass, and e) residual smoothness of mouth coating. Error bars represent the standard error of the mean. Cheeses included 2 full-fat cheeses (FF1 and FF2) that differed only in inclusion of a curd washing while making FF2, 1 reduced-fat (RF) cheese, and 2 low-fat cheeses (LF1 and LF2) that differed only in the starter culture used.

that these cheeses deformed more at a constant stress than FF cheeses. Brown et al. (2003) found a strong negative correlation between $\mathrm{J}_{\max }$ values and sensory firmness of cheeses with a higher $J_{\max }$ indicating a less firm texture (more on this in the discussion on correlations among sensory and mechanical terms). Within the linear region, the network structure of RF cheese behaved in a similar fashion to FF cheeses. Percentage recovery (an indication of elastic recovery) and retardation time (a measure of viscous relaxation) values did not show any clear differences in either age or fat content. This suggests that the overall viscoelasticity did not differ among cheeses.

\section{Fracture and Nonlinear Properties}

Nonlinear Curve Fitting. The section of the stressstrain curve between the linear viscoelastic region and fracture was designated as the "nonlinear" region; the differences in the shape of this region can be determined 


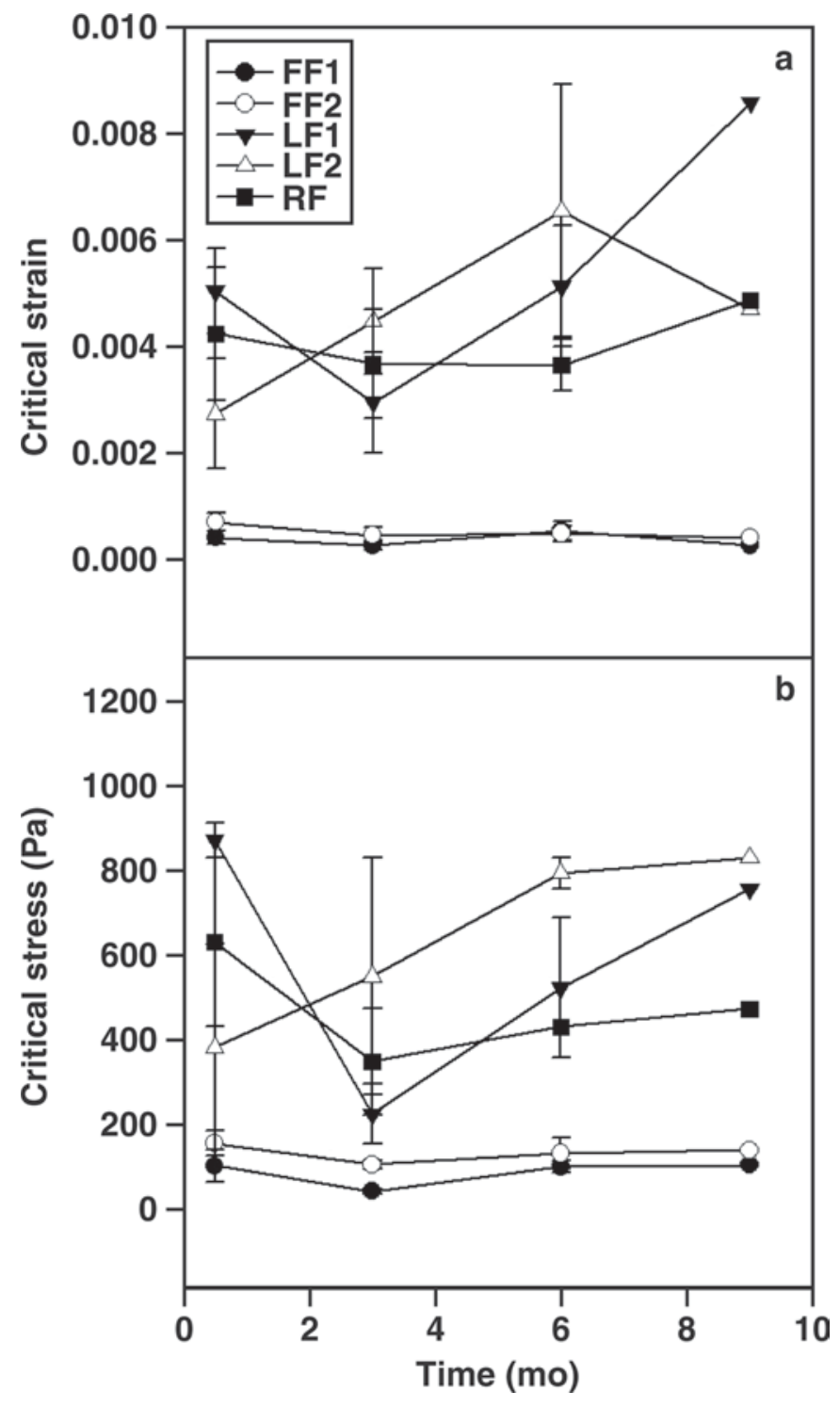

Figure 4. Critical a) strain and b) stress values for stress sweeps conducted at $10 \mathrm{~Hz}$. Error bars represent the standard error of the mean. Cheeses included 2 full-fat cheeses (FF1 and FF2) that differed only in inclusion of a curd washing while making FF2, 1 reduced-fat (RF) cheese, and 2 low-fat cheeses (LF1 and LF2) that differed only in the starter culture used.

using mathematical modeling. All cheeses showed a strain-weakening behavior in this region, in which an incremental increase in strain is associated with a decreased incremental stress (Figure 6a). Table 5 lists the $G_{\text {initial }}$ values determined from the stress strain curves as well as parameters from the BST model derived from a nonlinear curve-fitting algorithm. The nonlinear data showed no consistent aging effect in any of the cheeses, and $G_{\text {initial }}$ showed no discernable changes or trends across either time or fat content. This indicates that the stress-strain relationship at low levels of defor-

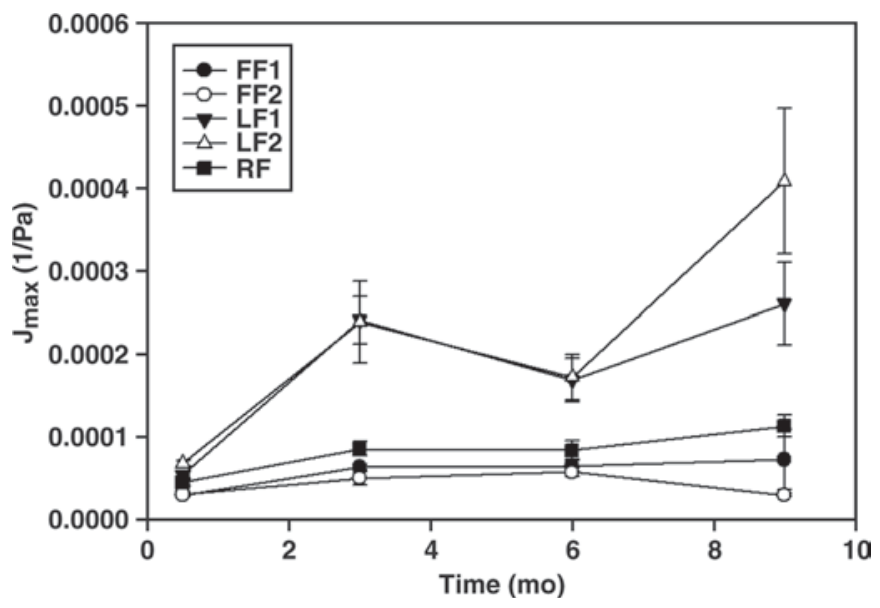

Figure 5. Maximum compliance $\left(\mathrm{J}_{\max }\right)$ from creep/recovery tests conducted at $150 \mathrm{~Pa}$. Error bars represent the standard error of the mean. Cheeses included 2 full-fat cheeses (FF1 and FF2) that differed only in inclusion of a curd washing while making FF2, 1 reduced-fat (RF) cheese, and 2 low-fat cheeses (LF1 and LF2) that differed only in the starter culture used.

mation (strain) was similar among all of the cheeses. The predicted values for $G_{B S T}$ were generally greater than the measured modulus $\left(G_{\text {initial }}\right.$; Table 5$)$. This was caused by the BST model overestimating the modulus at low strains (note deviation observed in Figure 6b). The predicted $G_{B S T}$ values for LF cheeses showed no real differences with relation to both fat content and aging time. In contrast to the modulus, the shape of the nonlinear stress strain relationship, governed by the shape factor $n$ in the BST model, was a better indicator of differences among cheeses in this region. The mathematical representation of the overall curve is represented by the shape factor $n$ in the BST model. A value of $n=2$ is indicative of an ideal elastic material, thus deviation from this gives an idea to the relative viscoelasticity, breakdown, or both (Barrangou et al., 2006a). The LF and RF cheeses had higher $n$ values, suggesting a more rubbery or elastic texture than their FF counterparts.

Torsional Fracture. Fracture stress, strain, and modulus (determined at $0.40 \mathrm{~s}^{-1}$ ) are presented in Figure 7. Although all cheeses showed a decrease in fracture stress (Figure 7a) over time, the LF cheeses had the largest change as they aged, whereas the FF cheeses exhibited a very small decrease. This may indicate that the lack of fat in the structure of the LF cheeses resulted in higher effect of proteolysis; this is consistent with the aging effect seen in LF creep/recovery terms. Fracture strain (Figure 7b) showed a small downward trend due to aging. Fracture strain did show differences due to fat content, with the FF cheeses grouped together and at lower fracture strains than the LF cheeses; this behav- 

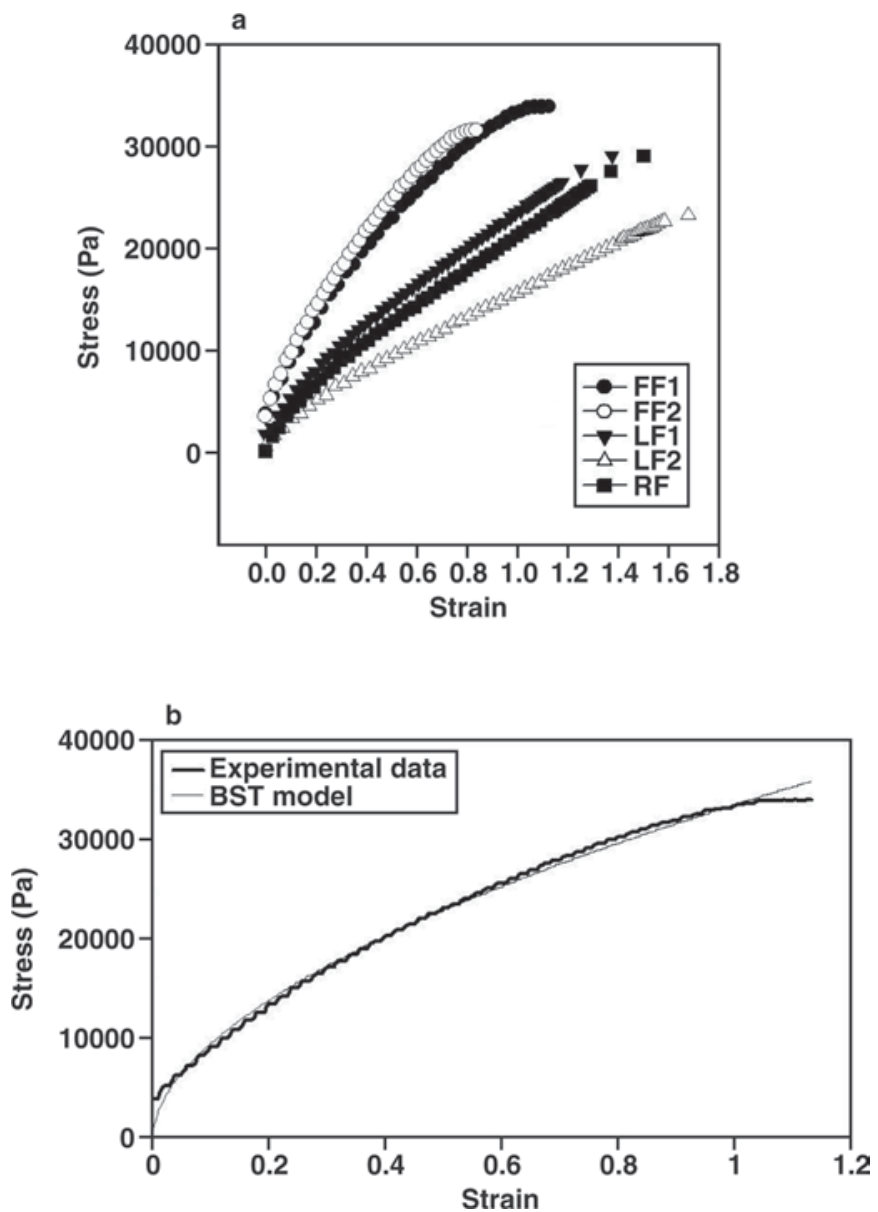

Figure 6. Stress-strain curves for a) Cheddar cheeses of varying fat contents at 6 mo of age; curves show every fifth data point improve clarity. Cheeses included 2 full-fat cheeses (FF1 and FF2) that differed only in inclusion of a curd washing while making FF2, 1 reduced-fat $(\mathrm{RF})$ cheese, and 2 low-fat cheeses (LF1 and LF2) that differed only in the starter culture used. b) Actual data and Blatz, Sharda, and Tschoegl (BST) equation fit for full-fat standard Cheddar cheese at 6 mo of age.

ior was also seen with the critical strain measurements. These trends mean that the FF cheeses were stronger (higher fracture stress) than the LF cheeses, the opposite of what was observed with sensory firmness. This is possibly because sensory firmness reflected the entire force-deformation relationship up to and including the fracture point. For example, the panelists may be responding to the nonlinear region before fracture (Figure 6a). Alternatively, panelists could be responding to a combined stress and strain sensation. Fracture modulus (Figure 7c), the fracture stress divided by the fracture strain, represents the combination of the stress and strain terms and thus provides one term that can relate the data from both. These data fit the sensory firmness trends better than fracture stress alone.

Other sensory texture studies have been conducted looking at more ideal food materials, such as gelatin

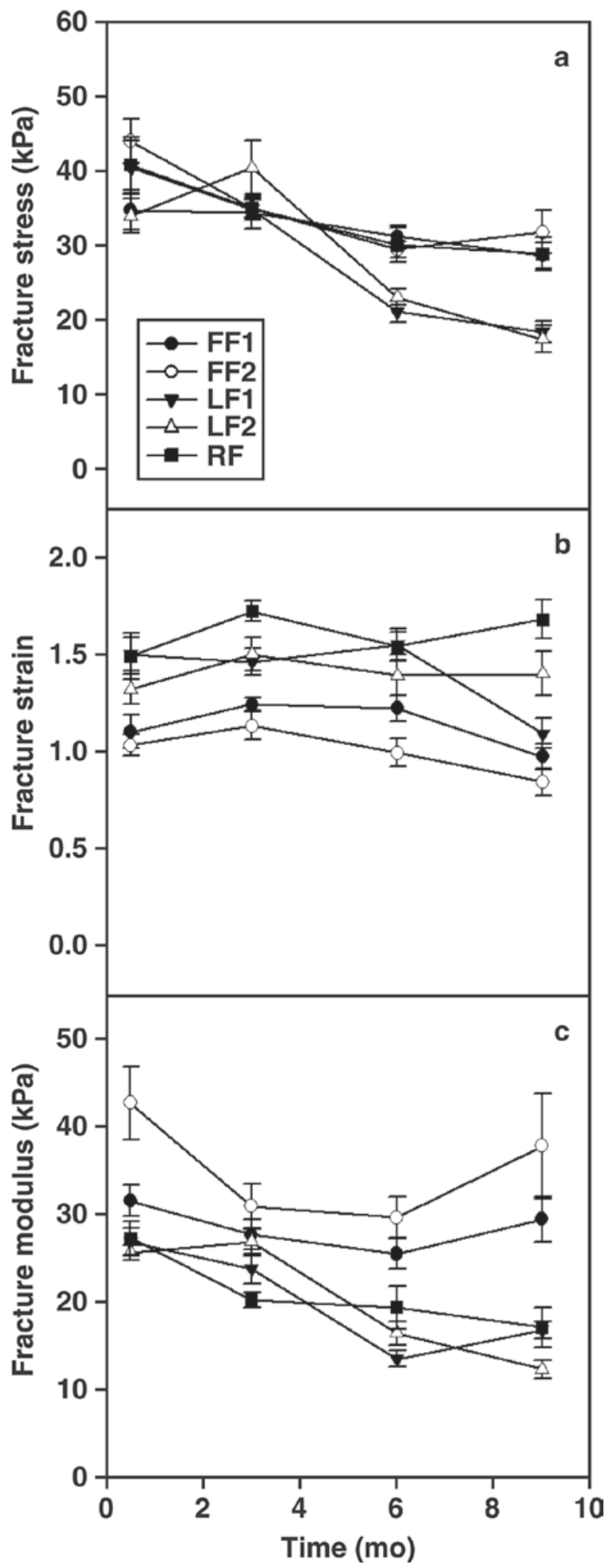

Figure 7. Torsional fracture a) stress, b) strain, and c) modulus for Cheddar cheese determined at a strain rate of $0.411 / \mathrm{s}$. Error bars represent the standard error of the mean. Cheeses included 2 fullfat cheeses (FF1 and FF2) that differed only in inclusion of a curd washing while making FF2, 1 reduced-fat (RF) cheese, and 2 low-fat cheeses (LF1 and LF2) that differed only in the starter culture used. 
Table 5. Nonlinear region values derived from the Blatz, Sharda, and Tschoegl (BST) equation $\left(G_{B S T}, n\right)$, and calculation of the initial slope $\left(G_{\text {initial }}\right)$ in Cheddar cheeses of varying fat contents

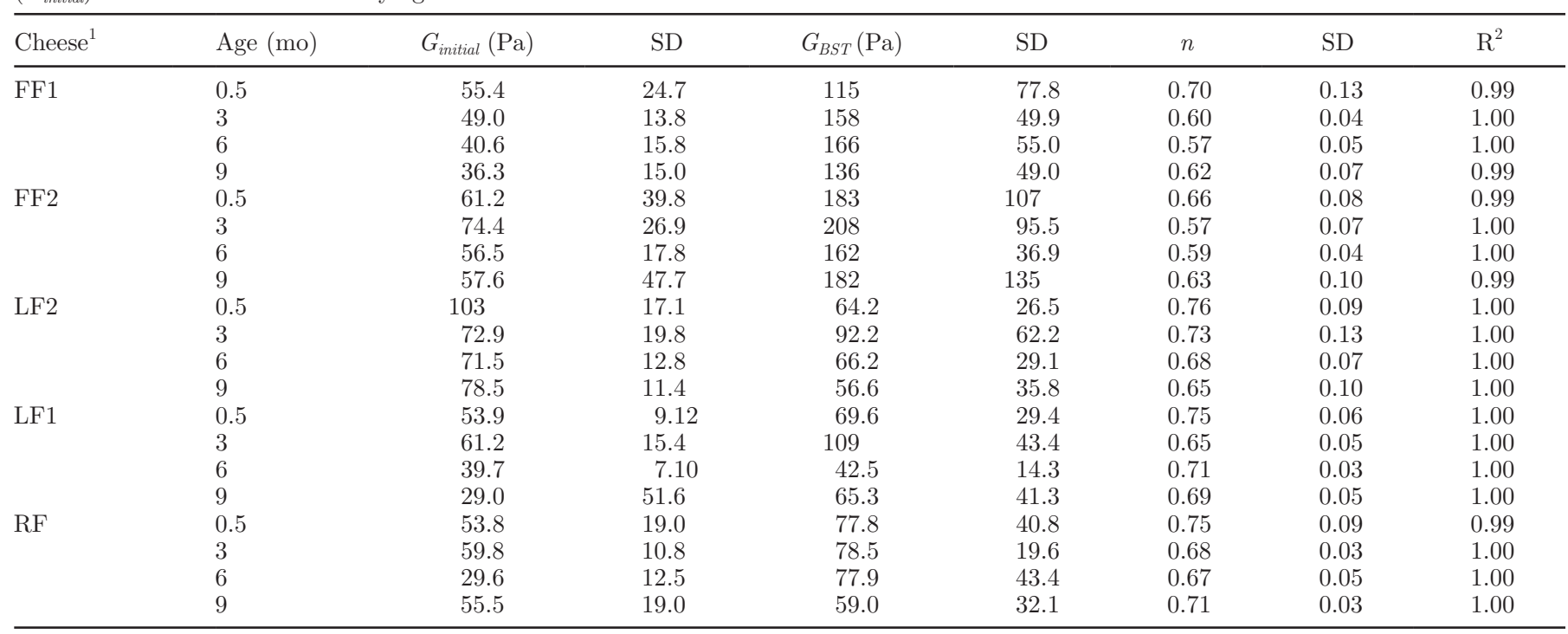

${ }^{1}$ Cheeses included 2 full-fat cheeses (FF1 and FF2) that differed only in inclusion of a curd washing while making FF2, 1 reduced-fat (RF) cheese, and 2 low-fat cheeses (LF1 and LF2) that differed only in the starter culture used.

and agarose, and the relationships among mechanical properties and sensory texture. In the case of agarose and agar gels, it was found that fracture properties were more related to the assessed sensory terms than the small-strain properties (Barrangou et al., 2006b,c). These materials are similar to cheeses in that they are soft solids but differ in stress-strain relationships, with agarose and agar gels showing strain hardening rather than strain weakening. In addition, weak spots or cracks are present in all materials and in gels made from agarose and agar; these cracks are few and small but still contribute to the failure of a material (van Vliet and Walstra, 1995). In the case of cheese, however, these cracks and imperfections are much larger and more numerous, affecting the way the material fractures. Therefore, the relationship between structure and sensory data seen in polymer gels is not always consistent with the relationship seen in cheese. Because of this, it is important to understand not only why the simpler, ideal systems work, but also the behavior of more complex structures such as cheese.

Because cheese is a viscoelastic material, fracture properties will show a time dependency because of the extent of viscous relaxations occurring during the testing. Strain rates were varied over 2 orders of magnitude $\left(0.04\right.$ to $\left.4.0 \mathrm{~s}^{-1}\right)$ to determine the time dependency. Because no aging-related trend was observed (data not shown), data for 6 mo aging are presented. All cheeses increased in fracture stress as strain rate increased (Figure 8a). This is typical behavior for a viscoelastic solid (vanVliet and Walstra, 1995) and has been observed for Mozzarella and Monterey Jack cheese by Brown et al. (2003), as well as Cheddar tested under different compression rates by Xiong et al. (2002). This rate effect was attributed, by vanVliet and Walstra (1995), to how bonds in a material "unzip." At low strain rates, the materials are allowed to unzip at a rate closer to the deformation rate, meaning less energy is needed to fracture a material, resulting in a lower fracture stress. Fracture strain is expected to increase or decrease with strain rate, depending on whether the energy dissipation is caused by frictional or viscous mechanisms, respectively (vanVliet and Walstra, 1995). Cheddar cheese does not follow a consistent trend so it either does not fit this model or shows a combined frictional/ viscous mechanism.

Surface Adhesion. Building on work done by Brown et al. (2003), adhesion testing was conducted in an attempt to find a rheological test that better related to sensory breakdown terms. Results for total adhesive force are presented in Figure 9. This property showed the same large initial change between 0.5 and 3 mo seen in many of the sensory results combined with a similar starting adhesive force for all the cheeses. After the 3 mo, the LF cheeses had more surface adhesion than the FF cheese, with the RF cheese falling in between the two. This trend follows the rheological tenets laid out by Dahlquist (1989), which involves materials with a storage modulus $\left(\mathbf{G}^{\prime}\right)$ less than $10^{5} \mathrm{~Pa}$ being considered pressure sensitive. Compliance $(\mathbf{J})$ is the inverse of $\mathrm{G}^{\prime}$, therefore $1 / \mathrm{J}_{\max }=\mathrm{G}^{\prime}$. In this study, $\mathrm{G}^{\prime}\left(1 / \mathrm{J}_{\max }\right)$ ranged from 2,500 to $20,000 \mathrm{~Pa}$, consistent with previous studies showing young cheeses to have $G^{\prime}$ values ranging between $10^{3}$ and $10^{4}$ (Brown et al., 2003). The 
viscoelastic nature of cheese makes the storage modulus variable according to the time scale of the test and temperature, and this will affect adhesion (Childs et al., 2007). Although the time scales between adhesive force and $\mathrm{J}_{\max }$ measurements are different, the relative differences among cheeses scale according to the Dahlquist criteria (Dahlquist, 1989). Low-fat cheeses, which had higher $\mathrm{J}_{\max }$ (lower $\mathrm{G}^{\prime}$ ) with the creep/recovery test, also had the highest adhesion area in this test. However, trends in sensory adhesion (Figure 3c) were opposite to those of mechanical adhesion. The reason for this difference was not apparent; however, pressuresensitive adhesion also involves surface energies of the 2 interacting materials (Hammond, 1989). Although surface energies were not assessed in this study, it is plausible that compositional factors dominate surface energies (Childs et al., 2007), explaining the differences in pressure-sensitive adhesion. In addition, the role of saliva in sensory adhesiveness is unknown.

\section{Correlation Among Sensory Texture Terms and Mechanical Properties}

As a framework to better understand the relationships among sensory and rheological tests, strain levels of the 3 rheologically defined regions are shown in Figure 10. The LVR is terminated at the point where critical stress and strain was determined. Within this region, forces and deformations are low so as to measure mechanical properties without altering the material. As the level of strain increases to the critical point and nonlinear region (Figure 10), progressively more damage is done to the structure or nonlinear changes detected. Correlation among sensory terms and $\mathrm{J}_{\max }$, critical stress and strain, adhesion, $G_{B S T}$, and $n$ are presented at $0.5,3,6$, and 9 mo to show the progression of these properties with age (Table 6 ). Maximum compliance was positively correlated with sensory hand and mouth firmness. This is most likely related to the effect of the fat, which acts to firm up the structure, rather than a true correlation with mouth evaluation of firmness, because sensory firmness and $\mathrm{J}_{\max }$ should have an inverse relationship, as was observed by Brown et al. (2003). Likewise, because $\mathrm{J}_{\max }$ is measured at nondestructive forces and deformations, the high correlations with breakdown terms most likely reflect compositional differences rather than an association with specific textural properties. As the cheese aged, critical stress and strain values became increasingly correlated to breakdown sensory terms. Negative correlations were observed between both critical stress and critical strain and the breakdown terms degree of breakdown, cohesiveness, adhesiveness, smoothness of mass, and residual smoothness of mouth coating at 6 and 9 mo of aging; as seen in Figure 4,

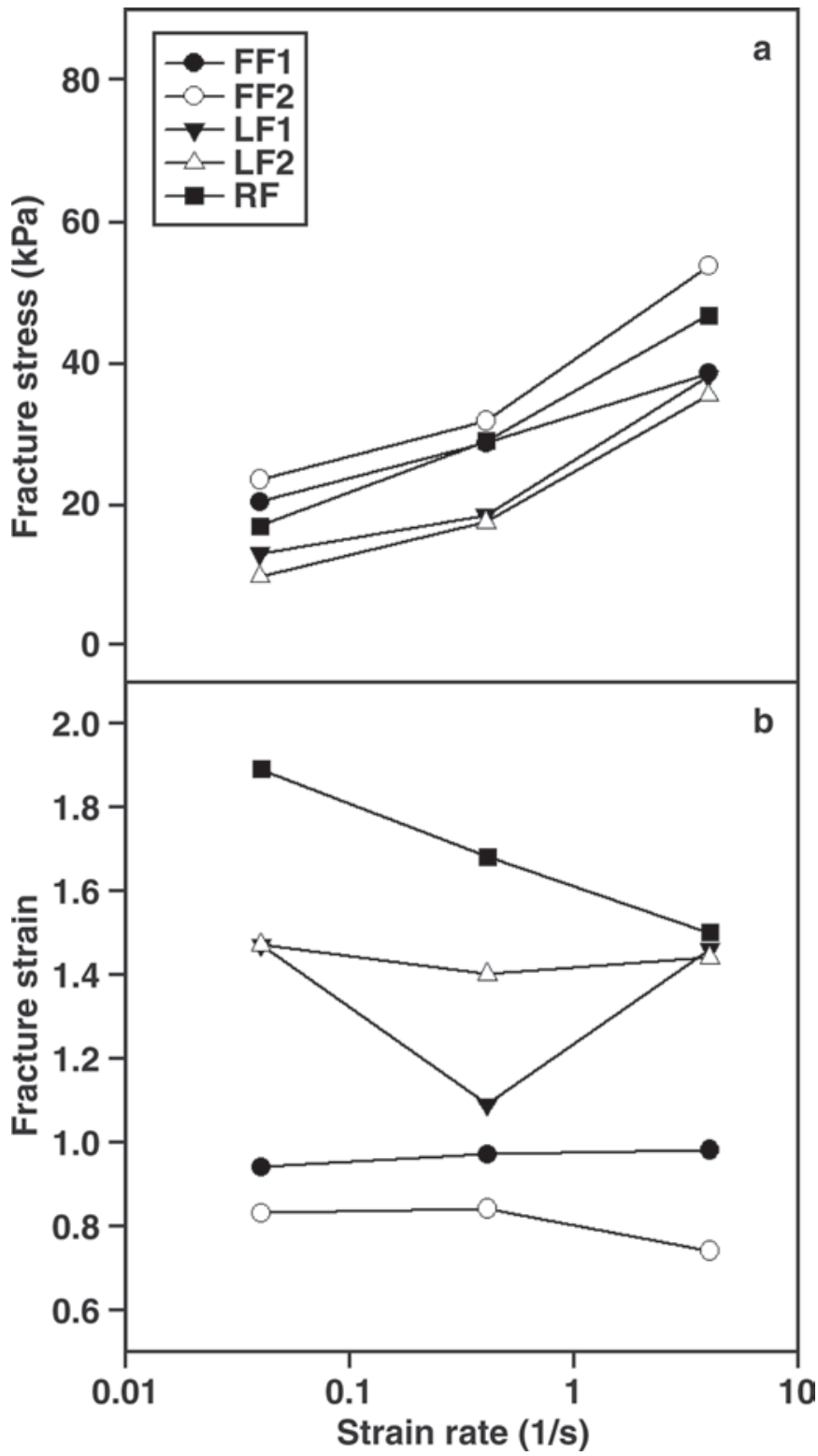

Figure 8. Effect of strain rate on torsional fracture a) stress and b) strain of Cheddar cheese at 9 mo. Cheeses included 2 full-fat cheeses (FF1 and FF2) that differed only in inclusion of a curd washing while making FF2, 1 reduced-fat (RF) cheese, and 2 low-fat cheeses (LF1 and LF2) that differed only in the starter culture used.

this is mainly due to a clear differentiation between FF and the other cheeses. Critical strain also correlated with hand springiness and hand rate of recovery terms. The positive correlations with firmness terms and negative correlations with breakdown terms for critical stress and strain are logical based on changes in cheese structure and suggest that changes in extent of the linear viscoelastic region are related to sensory texture. The initial modulus determined from the BST model $\left(G_{B S T}\right)$ had a consistent negative correlation with 


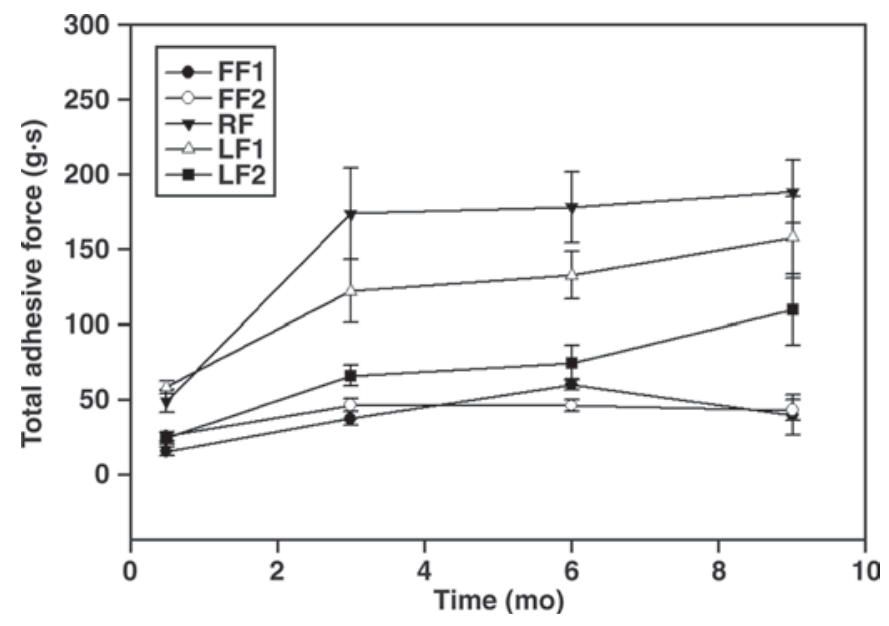

Figure 9. Total adhesive force for Cheddar cheese. Error bars represent the standard error of the mean. Cheeses included 2 full-fat cheeses (FF1 and FF2) that differed only in inclusion of a curd washing while making FF2, 1 reduced-fat (RF) cheese, and 2 low-fat cheeses (LF1 and LF2) that differed only in the starter culture used.

some firmness terms (hand springiness, hand rate of recovery, and mouth firmness), but because the correlation was negative, it is most likely related to fat content and not sensory detection of this mechanical property. The shape factor $(n)$ showed strong negative correlations to the sensory breakdown terms, that was most apparent at 6 and 9 mo. As this value decreased, it indicated more strain weakening in the cheese that could result in a greater degree of breakdown in the mouth resulting in higher smoothness of mass, residual smoothness of mouth coating, cohesiveness, and adhesiveness. This correlation and plausible explanation suggests that the shape of the stress-strain curve, as seen in Figure 6a, reflects changes in cheese structure that are linked to key sensory texture terms. Similarly, the degree of energy loss, which is related to the strain weakening behavior seen in the cheese, occurring during deformation of mixed whey protein-polysaccharide gels is highly correlated with sensory texture (van den Berg et al., 2008).

Adhesion was highly negatively correlated with the sensory breakdown terms at 6 and 9 mo of age. At first thought, this may seem odd because adhesiveness and instrumental adhesion (area under adhesion curve) are both measuring adhesion between surfaces, and a positive correlation would be expected. One possibility why this relationship was negative is that interactions in the mouth, which involve saliva and changing temperature, are different from the direct instrumental measure of adhesion. This theory was proposed by Steiner et al. (2003) concerning sensory perception of adhesion and instrumental tack of caramel. Another possible explanation for this inverse relationship is the difference in the unchewed adhesion (adhesion between the probe and unchewed cube) and sensory breakdown adhesion (adhesion between particles coated with saliva and mouth surfaces). There was also a positive correlation between adhesion area and the sensory firmness terms, which contradicts the Dahlquist criterion discussed earlier. This could mean that adhesion is surface energydependent, which has been shown in other cheeses such as Mozzarella (Childs et al., 2007).

\section{Correlation Between Sensory and Fracture Properties}

Correlations among fracture at the 3 strain rates and sensory terms were determined at $0.5,3,6$, and $9 \mathrm{mo}$

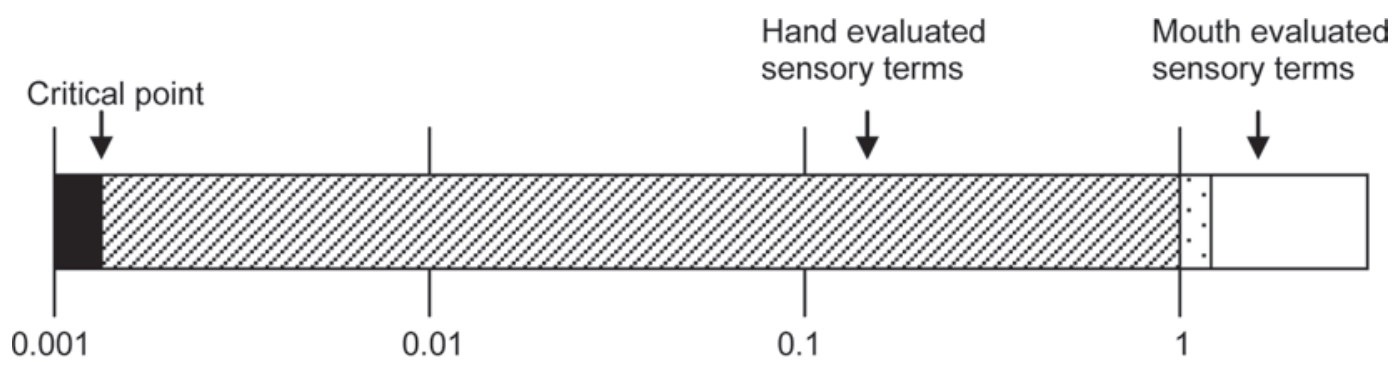

Strain

Linear viscoelastic region (creep/recovery test)

$\square$ Non-linear region (non-linear curve fitting)

\section{Fracture of material (torsional fracture test)}

Figure 10. Strain representation on a logarithmic scale of the rheological testing compared with sensory texture test methods. 
Table 6. Correlation analysis for maximum compliance $\left(\mathrm{J}_{\max }\right)$, area under adhesion curve, critical stress and strain, initial slope of the fracture curve $\left(G_{B S T}\right)$, and shape factor constant $(n)$ of the nonlinear region with sensory texture terms by month of aging ${ }^{1}$

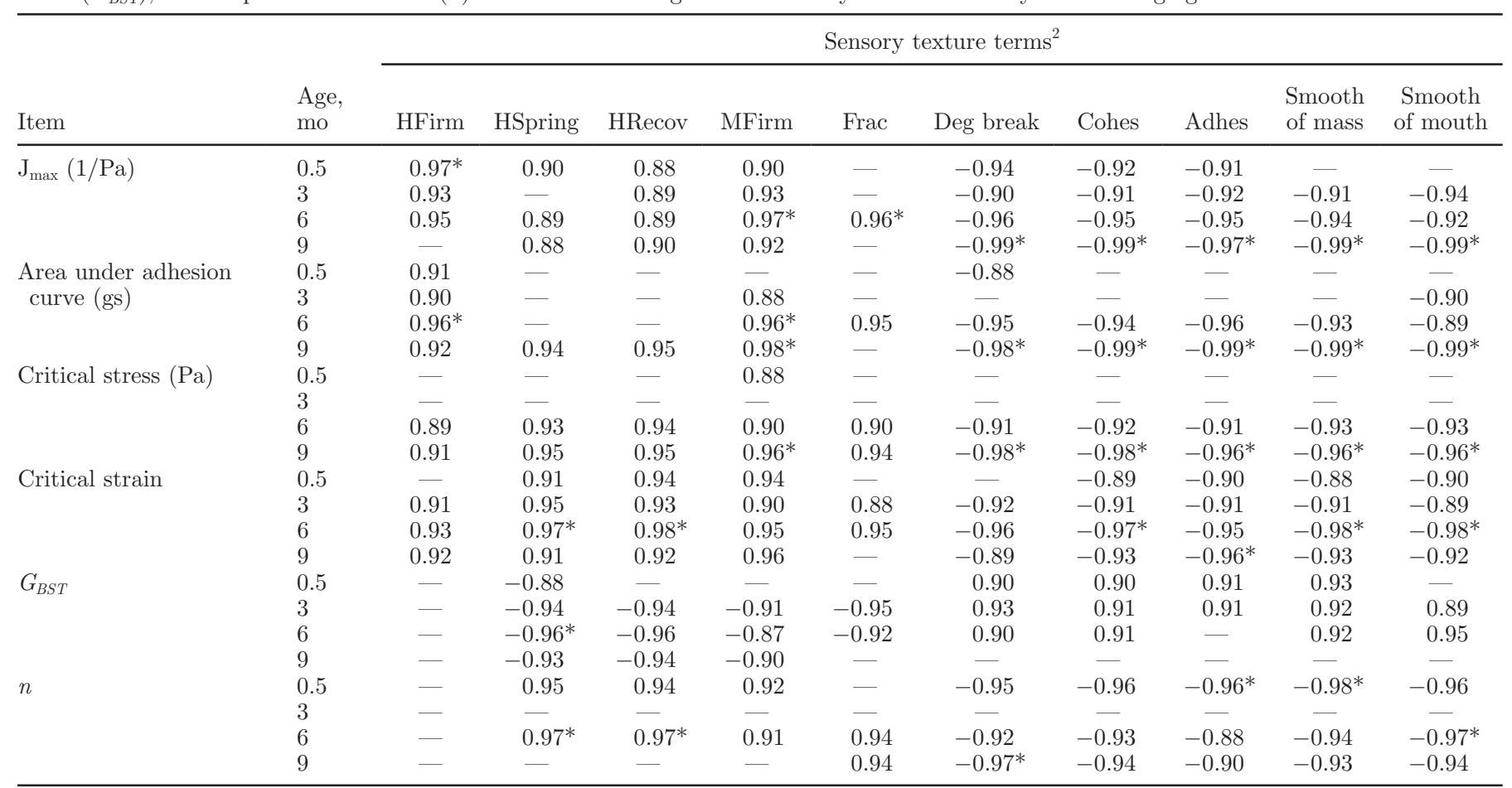

${ }^{1}$ Values in table indicate $P<0.05 ; *$ indicates $P<0.01 ;$ indicates $P>0.05$.

${ }^{2}$ HFirm $=$ hand firmness; HSpring $=$ hand springiness; HRecov $=$ hand rate of recovery; MFirm $=$ first-bite firmness; Frac $=$ first-bite fracturability; Deg break = degree of breakdown; Cohes = cohesiveness; Adhes = adhesiveness; Smooth of mass = smoothness of mass; and Smooth of mouth $=$ residual smoothness of mouth coating.

(Table 7). Across all strain rates and aging times, correlations were consistently seen with hand and mouth firmness terms, springiness, and hand rate of recovery, and most of the breakdown terms. Fracture modulus was the mechanical property most correlated with sensory properties. Fracture modulus was negatively correlated with hand and first-bite terms and positively correlated with the breakdown terms. Correlations involving fracture properties revealed some unexpected results. Fracture stress values showed negative relationships with the hand and first-bite terms. This means that a decrease in fracture stress translates into a firmer cheese as perceived by sensory analysis, which is not logical. Likewise, fracture modulus (fracture stress divided by fracture strain) also shows this negative relationship. Inspection of the stress-strain curves (Figure 6a) shows that the FF cheeses had higher fracture stress, lower fracture strain, and generally a greater strain weakening (lower $n$, Table 4) than LF or RF cheeses. It is possible that the strain-weakening behavior is dominating the sensory detection of firmness and not just the final point of fracture. This possibility needs to be evaluated further. The negative correlations among fracture modulus and hand springiness and hand rate of recovery have been noted by Brown et al. (2003) and Carunchia Whetstine et al. (2007). Breakdown terms were positively correlated with fracture stress and negatively correlated with fracture strain; this means that as cheeses became more brittle, the degree of breakdown increased. The lowest strain rate showed the highest number of correlations between the fracture and sensory terms. Logic suggests that properties measured at the highest strain rate, which are closer to the strain rates seen in the mouth during chewing, would correlate better with the sensory terms. However, as seen in this investigation and that of Xiong et al. (2002), lower strain rates correlate better with sensory hardness of cheese.

\section{CONCLUSIONS}

Overall, FF cheeses behaved similarly to each other, regardless of make procedure as did the 2 LF cheeses, which were distinguished only by starter culture. The $\mathrm{RF}$ cheeses fell in between the FF and LF behaviors. Many of the changes seen in all the cheeses occurred in the first 3 mo of aging. Rheological properties determined at strains causing initial yielding (critical stress and strain), along with the shape of the stress-strain curve $(n)$, showed the highest correlations that had a plausible link between cheese structure and sensory tex- 
Table 7. Correlation analysis for fracture properties with sensory texture terms by month of aging ${ }^{1}$

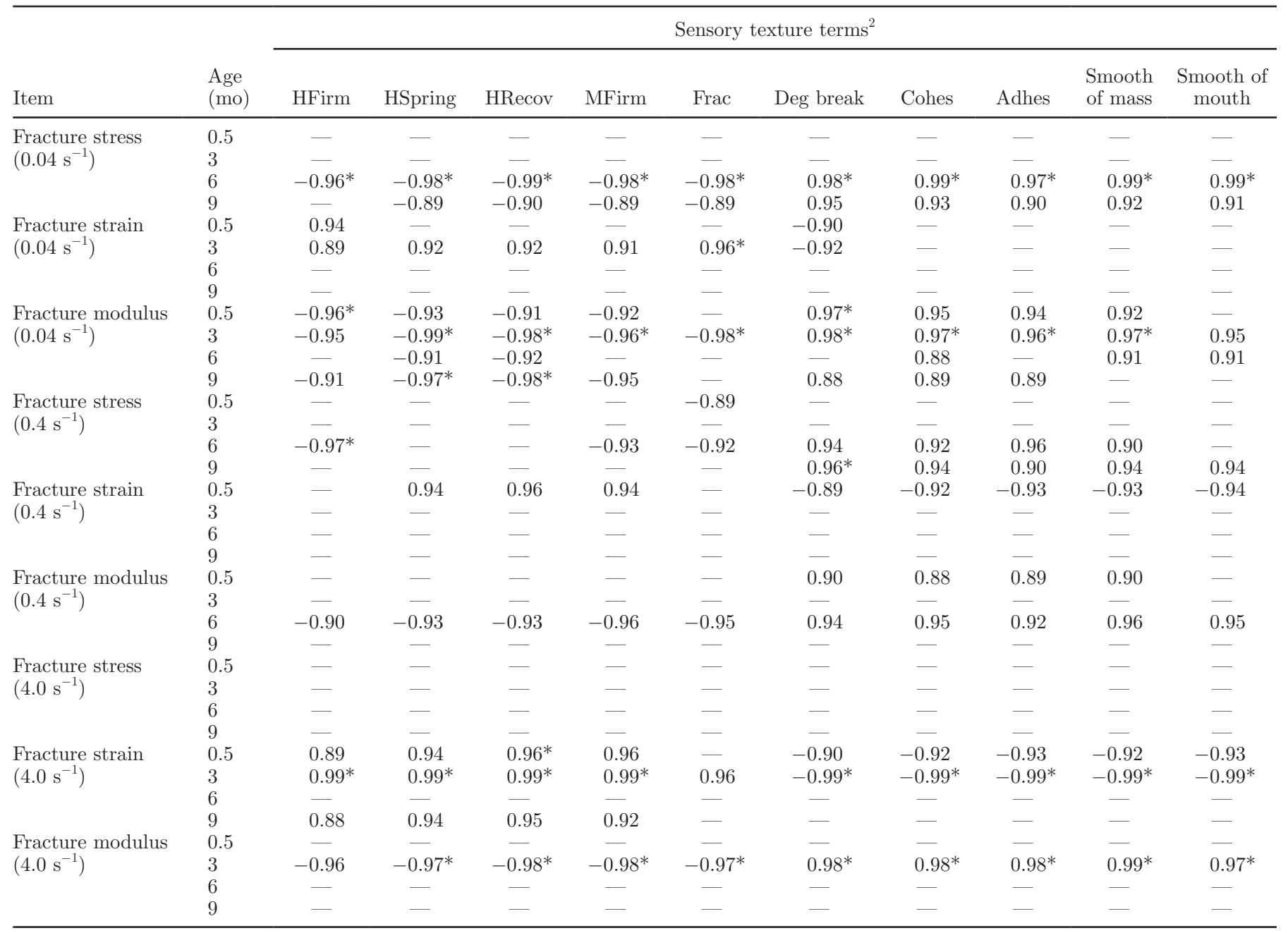

${ }^{1}$ Values in table indicate $P<0.05 ; *$ indicates $P<0.01 ;$ - indicates $P>0.05$.

${ }^{2}$ HFirm $=$ hand firmness; HSpring $=$ hand springiness; HRecov $=$ hand rate of recovery; MFirm $=$ first-bite firmness; Frac $=$ first-bite fracturability; Deg break = degree of breakdown; Cohes = cohesiveness; Adhes = adhesiveness; Smooth of mass = smoothness of mass; and Smooth of mouth $=$ residual smoothness of mouth coating.

ture terms, especially terms associated with breakdown during chewing. These breakdown terms, which showed the most sensory separation between cheeses, appear to be one of the major sensory contributors to the texture differences in the cheeses. These terms, although correlated with aspects of the nonlinear region and critical stress and strain, do not show the same high levels of correlation with rheological tests that firmness and springiness terms show. The combined results suggest that when Cheddar cheese structure can be represented as varying amounts of fat in a compositionally similar protein network (i.e., constant moisture:protein ratio), an increase in fat content causes a stiffening of the network (lower $\mathrm{J}_{\max }$ ), and at the same time provides weak spots in the form of fat-protein interfaces. The weak spots cause more strain weakening during deformation (i.e., lower $n$ ) and lower fracture strain, and produce smaller particles during fracture. This results in less firmness and a higher degree of breakdown during chewing.

\section{ACKNOWLEDGMENTS}

This is paper no. FSR-09-01 of the Journal Series of the Department of Food, Bioprocessing and Nutrition Science, North Carolina State University (Raleigh). Support from the North Carolina Agricultural Research Service, Dairy Management Inc., and Southeast Dairy Foods Research Center are gratefully acknowledged. The use of trade names in this publication does not imply endorsement by the North Carolina Agricultural Research Service of the products named nor criticism of 
similar ones not mentioned. This project was part of a collaboration with Utah State University, and we thank the Department of Nutrition and Food Sciences at Utah State for the production and aging of the cheeses. The technical support of Michelle Yates and Paige Luck of North Carolina State University (Raleigh) is gratefully acknowledged.

\section{REFERENCES}

Banks, J. M. 2007. What general factors affect the texture of hard and semi-hard cheeses? Pages 200-201 in Cheese Problems Solved. P. L. H. McSweeney, ed. Woodhead, Boca Raton, FL.

Barrangou, L. M., C. R. Daubert, and E. A. Foegeding. 2006a. Textural properties of agarose gels. I. Rheological and fracture properties. Food Hydrocoll. 20:184-195.

Barrangou, L. M., M. A. Drake, C. R. Daubert, and E. A. Foegeding. 2006b. Textural properties of agarose gels. II. Relationships between rheological properties and sensory texture. Food Hydrocoll. 20:196-203.

Barrangou, L. M., M. A. Drake, C. R. Daubert, and E. A. Foegeding. 2006c. Sensory texture related to large-strain rheological properties of agar/glycerol gels as a model food. J. Texture Stud. 37:241262

Blatz, P. J., S. C. Sharda, and N. W. Tschoegl. 1974. Strain energy function for rubberlike materials based on a generalized measure of strain. Trans. Soc. Rheol. 18:145-161.

Brown, J. A., E. A. Foegeding, C. R. Daubert, M. A. Drake, and M. Gumpertz. 2003. Relationships among rheological and sensorial properties of young cheese. J. Dairy Sci. 86:3054-3067.

Bryant, A., Z. Ustunol, and J. Steffe. 1995. Texture of Cheddar cheese as influenced by fat reduction. J. Food Sci. 60:1216-1236.

Carunchia Whetstine, M. E., P. J. Luck, M. A. Drake, E. A. Foegeding, and P. D. Gerard. 2007. Characterization of flavor and texture development within large $(291 \mathrm{~kg})$ blocks of Cheddar cheese. J. Dairy Sci. 90:3091-3109.

Casiraghi, E., M. Lucisano, and C. Pompei. 1989. Correlation among instrumental texture, sensory texture and chemical composition of five Italian cheeses. Ital. J. Food Sci. 1:53-64.

Childs, J. L., C. R. Daubert, L. Stefanski, and E. A. Foegeding. 2007. Factors regulating cheese shreddability. J. Dairy Sci. 90:21632174

Dahlquist, C. A. 1989. Creep. Pages 97-114 in Handbook of Pressure Sensitive Adhesive Technology. 2nd ed. D. Satas, ed. Van Nostrand Reinhold, New York, NY.

Diehl, K. C., and D. D. Hamann. 1979. Structural failure in selected raw fruits and vegetables. J. Texture Stud. 10:371-400.

Drake, M., P. Gerard, and G. Civille. 1999a. Ability of hand evaluation versus mouth evaluation to differentiate texture of cheese. J. Sens. Stud. 14:425-441.

Drake, M. A., P. D. Gerard, V. D. Troung, and C. R. Daubert. 1999b. Relationship between instrumental and sensory measurements of cheese texture. J. Texture Stud. 30:451-476.

Drake, M. A., W. Herrett, T. D. Boylston, and B. G. Swanson. 1995. Sensory evaluation of reduced fat cheeses. J. Food Sci. 60:898901

Drake, M. A., and B. G. Swanson. 1995. Reduced- and low-fat cheese technology: A review. Trends Food Sci. Technol. 6:366-369.

Drake, M. A., V. D. Truong, and C. R. Daubert. 1999c. Rheological and sensory properties of reduced-fat processed cheeses containing lecithin. J. Food Sci. 64:744-747.

Foegeding, E. A. 2007. Rheology and sensory texture of biopolymer gels. Curr. Opin. Colloid Interface Sci. 12:242-250.

Foegeding, E. A., J. Brown, M. A. Drake, and C. R. Daubert. 2003. Sensory and mechanical aspects of cheese texture. Int. Dairy J. 13:585-591.
Foegeding, E. A., and M. A. Drake. 2007. Sensory and mechanical properties of cheese texture. J. Dairy Sci. 90:1611-1624.

Gwartney, E. A., E. A. Foegeding, and D. K. Larick. 2002. The texture of commercial full-fat and reduced-fat cheese. J. Food Sci. $67: 812-816$

Hamann, D. D. 1983. Structural failure in solid foods. Pages 351-383 in Physical Properties of Foods. M. Peleg and E. B. Bagley, ed. AVI, Westport, Connecticut.

Hamilton, J., B. Knox, D. Hill, and H. Parr. 2000. Reduced fat products consumer perceptions and preferences. Br. Food J. 102:494-506.

Hammond, F. H. 1989. Tack. Pages 38-60 in Handbook of Pressure Sensitive Adhesive Technology. 2nd ed. D. Satas, ed. Van Nostrand Reinhold, New York.

Hort, J., G. Grys, and J. Woodman. 1997. The relationships between the chemical, rheological and textural properties of cheddar cheese. Lait 77:587-600.

Hort, J., and G. L. Grys. 2000. Rheological models of cheddar cheese texture and their application to maturation. J. Texture Stud. $31: 1-24$.

Jack, F. R., J. R. Piggott, and A. Paterson. 1993. Relationships between rheology and composition of Cheddar cheese and texture as perceived by consumers. Int. J. Food Sci. Technol. 28:293302.

Johnson, M. E., and C. M. Chen. 1995. Technology of manufacturing reduced-fat cheddar cheese. Pages $331-337$ in Chemistry of Structure-Function Relationships in Cheese. E. L. Malin and M. H. Tunick, ed. Plenum Press, New York, NY.

Lawrence, R. C., L. K. Creamer, and J. Gilles. 1987. Texture development during cheese ripening. J. Dairy Sci. 70:1748-1760.

Lee, C., E. Imoto, and C. Rha. 1978. Evaluation of cheese texture. J. Food Sci. 43:1600-1605.

Mistry, V. 2001. Low fat cheese technology. Int. Dairy J. 11:413422.

Nadai, A. 1937. Plastic behavior of metals in the strain-hardening range. Part I. J. Appl. Physics 8:205-213.

Sandrou, D. K., and I. S. Arvanitoyannis. 2000. Low-fat/calorie foods: Current state and perspectives. Crit. Rev. Food Sci. Nutr. 40:427-447.

Scott, R. 1986. Cheesemaking Practice. 2nd ed. Elsevier Applied Science Publishers, New York, NY.

Steffe, J. F. 1996. Rheological Methods in Food Process Engineering. Freeman Press, East Lansing, MI.

Steiner, A. E., E. A. Foegeding, and M. Drake. 2003. Descriptive analysis of caramel texture. J. Sens. Stud. 18:277-289.

Truong, V. D., and C. R. Daubert. 2000. Comparative study on large strain methods in assessing failure characteristics of selected food gels. J. Texture Stud. 31:335-353.

Tunick, M. H., E. J. Nolan, J. J. Shieh, J. J. Basch, M. P. Thompson, B. E. Maleeff, and V. H. Holsinger. 1990. Cheddar and Cheshire cheese rheology. J. Dairy Sci. 73:1671-1675.

van den Berg, L., A. L. Carolas, T. van Vliet, E. van der Linden, M. A. J. S. van Boekel, and F. van de Velde. 2008. Energy storage controls crumbly perception in whey proteins/polysaccharide mixed gels. Food Hydrocoll. 22:1404-1417.

van Vliet, T., and P. Walstra. 1995. Large deformation and fracture behavior of gels. Faraday Discuss. 101:359-370.

Visser, J. 1991. Factors affecting the rheological and fracture properties of hard and semi-hard cheese. Pages 49-61 in Rheological and Fracture Properties of Cheese. P. Walstra, ed. International Dairy Federation, Brussels, Belgium.

Wells, H. F., and J. C. Buzby. 2007. Americans' Dairy Consumption Below Recommendations. Amberwaves, USDA/ERS, Washington, DC.

Xiong, R., J.-F. Meullenet, J. A. Hankins, and W. K. Chung. 2002. Relationship between sensory and instrumental hardness of commercial cheeses. J. Food Sci. 67:877-883.

Yates, M. D., and M. A. Drake. 2007. Texture properties of gouda cheese. J. Sens. Stud. 22:493-506. 